\title{
Mental health, psychotropic medication use, and menstrual cycle characteristics
}

This article was published in the following Dove Press journal:

Clinical Epidemiology

\author{
Yael I Nillni ${ }^{1,2}$ \\ Amelia K Wesselink ${ }^{3}$ \\ Elizabeth E Hatch ${ }^{3}$ \\ Ellen M Mikkelsen ${ }^{4}$ \\ Jaimie L Gradus ${ }^{1-3}$ \\ Kenneth J Rothman ${ }^{3,5}$ \\ Lauren A Wise ${ }^{3}$
}

'Department of Psychiatry, Boston University School of Medicine, Boston, MA, USA; ${ }^{2}$ National Center for PTSD, Women's Health Sciences Division at VA Boston Healthcare System, Boston, MA, USA; ${ }^{3}$ Department of Epidemiology, Boston University School of Public Health, Boston, MA, USA; ${ }^{4}$ Department of Clinical Epidemiology, Aarhus University, Aarhus, Denmark; ${ }^{5}$ RTI Health Solutions, Research Triangle, NC, USA
Correspondence: Yael I Nillni Boston University School of Medicine and National Center for PTSD at VA Boston Healthcare System, 150 South Huntington Ave. (II6B-3), Boston, MA 02130, USA

Tel +l 8573644637

Fax +l 8573644515

Email ynillni@bu.edu
Purpose: To examine the influence of depressive symptoms, perceived stress, and psychotropic medication use on prospectively assessed menstrual cycle regularity and length.

Participants and methods: Data were obtained from Pregnancy Study Online, a prospective cohort study of pregnancy planners from North America. At baseline, women reported information on demographics, anthropometrics, lifestyle, and medical history, including their use of psychotropic medications. They also completed the Major Depression Inventory and the Perceived Stress Scale. Every 8 weeks for up to 12 months or until conception, women completed follow-up questionnaires to assess changes in psychotropic medication use, perceived stress, and menstrual cycle characteristics (ie, cycle regularity and length). Women who completed at least one follow-up questionnaire from 2013 to $2018(n=3,346)$ were included in the primary analyses. A total of 5,439 women were included in secondary analyses utilizing baseline data only. Primary analyses estimated prevalence ratios (PR) and 95\% CIs using log-binomial regression models. Results: Women with severe depressive symptoms at baseline, regardless of psychotropic medication use, had an $80 \%$ greater prevalence of irregular cycles during follow-up than women with no or low depressive symptoms $(\mathrm{PR}=1.80,95 \% \mathrm{CI}=1.48-2.19)$. Perceived stress was also associated with the prevalence of irregular cycles during follow-up ( $\mathrm{PR}=1.33,95 \%$ $\mathrm{CI}=1.14-1.55)$. Psychotropic medication use was not appreciably associated with menstrual characteristics after controlling for history of diagnosed depression and/or anxiety. Depressive symptoms, perceived stress, and psychotropic medication use showed little association with menstrual cycle length.

Conclusion: Higher levels of depressive symptoms and perceived stress were associated with irregular menstrual cycles, but not appreciably associated with menstrual cycle length. Use of psychotropic medications was not meaningfully associated with cycle regularity or length.

Keywords: depression, menstrual cycle irregularity, perceived stress, psychotropic medication

\section{Introduction}

Menstrual function has a central role in women's physical and reproductive health. Menstrual disturbances have been associated with adverse health outcomes, including increased risks for coronary heart disease, ${ }^{1}$ type 2 diabetes mellitus, ${ }^{2}$ cardiometabolic disease ${ }^{3}$ and reduced fecundability. ${ }^{4,5}$ Therefore, it is important to identify factors associated with menstrual cycle dysfunction.

Depression and anxiety-related disorders are among the leading causes of years of life lost due to premature mortality or disability in the USA. ${ }^{6}$ There is a $2: 1$ female-tomale ratio in the prevalence of these disorders. ${ }^{7}$ Depression and anxiety disorders are associated with dysregulation of the hypothalamic-pituitary-adrenal axis (HPA), ${ }^{8-10}$ 
which can delay or impede the luteinizing hormone (LH) surge ${ }^{11}$ and impair ovarian function. Cross-sectional studies have found associations between depression and irregular menses, painful and heavier menses, and anovulation. ${ }^{3,12,13} \mathrm{In}$ a prospective cohort study, high perceived stress levels were associated with an increased probability of anovulation. ${ }^{14}$ Finally, some psychotropic medications (eg, valproate) can induce polycystic ovary syndrome, ${ }^{15,16}$ a condition associated with menstrual cycle irregularities. These findings indicate that mental health symptoms and their treatments may adversely affect menstrual function.

Few studies have prospectively examined the influence of mental health on menstrual cycle characteristics, or have considered both the individual and joint effects of depressive symptoms, perceived stress, and psychotropic medication use on cycle irregularity. The present study examines prospectively the association between current depressive symptoms, perceived stress, and psychotropic medication use and menstrual cycle regularity and length. Secondary analyses examined the association between these factors and menstrual bleed length and heaviness of menstrual flow.

\section{Participants and methods Study design}

Pregnancy Study Online (PRESTO) is a web-based prospective cohort study of pregnancy planners from the USA and Canada. The study is described in greater detail elsewhere. ${ }^{17}$ Briefly, eligible women were of age $21-45$ years, in a stable relationship with a male partner, not pregnant, and not using contraception or fertility treatments.

Participants completed an online baseline questionnaire, which assessed demographics, medical and reproductive history, and lifestyle habits. Follow-up questionnaires were administered every 8 weeks for up to 12 months or until reported conception to collect updated information on menstrual cycle characteristics, lifestyle and behavioral factors, and pregnancy status. Given that some women became pregnant and others were lost to follow-up at different time points, the number of follow-up questionnaires completed varied among study participants. The study was approved by the Institutional Review Board of Boston Medical Center, and all participants provided informed consent online.

\section{Assessment of menstrual cycle characteristics}

Information on current cycle regularity and length was assessed at baseline and at each follow-up questionnaire, in which women were asked, "Has your period been regular (regular in a way that you can usually predict when the next period will start)?" On the baseline questionnaire only, women were given the option to select "cannot say because I was taking hormones most of the time." On all questionnaires, women who reported regular menses were asked, "How long was your most recent cycle (days)?"

Data on menstrual bleed length and heaviness of flow were assessed on the baseline questionnaire only, where women were asked for their typical bleed length (ie, number of days of bleeding, not spotting) and total amount of menstrual flow (light: $\leq 10$ pads/tampons per menses, moderate: 11-20 pads/tampons per menses, moderate/heavy: 21-30 pads/tampons per menses, and heavy: $>30$ pads/tampons per menses).

\section{Assessment of mental health}

On the baseline questionnaire, participants reported depressive symptoms experienced during the previous 2 weeks via the 12-item Major Depression Inventory (MDI; possible range of scores 0-50). The MDI, a measure of depressive symptoms, has been validated in population-based studies and in patient populations, ${ }^{18-20}$ demonstrating acceptable sensitivity (0.86) and specificity (0.86) when compared with clinician-diagnosed major depression..$^{20}$ We used standard scoring criteria to categorize depression severity (i.e., none/ low $[<20]$, mild [20-24], moderate [25-29], severe [ $\geq 30]) .{ }^{21}$

On the baseline and follow-up questionnaires, participants reported the degree to which they perceived their lives as stressful in the past month via the 10-item Perceived Stress Scale (PSS; possible range of scores 0-40). Psychometric studies demonstrate that the PSS-10 reliably captures recent, but not chronic, stress and is highly correlated with acute physical symptoms and healthcare utilization. ${ }^{22-24}$ Scores were categorized into four groups based on the distribution in our cohort $(<15,15-19,20-24$, and $\geq 25)$.

History of depression and anxiety diagnoses were assessed on the baseline questionnaire using two separate questions (ie, "Have you ever been diagnosed with depression?" and "Have you ever been diagnosed with anxiety/panic disorder?”).

\section{Assessment of psychotropic medication use}

Participants provided information about medication use for depression, anxiety, and other conditions (eg, sleep disorders) at baseline. Specifically, participants reported which medications they had ever used, for how long they used them, the name of the medication taken for the longest and most recently, and whether they had used any medication in the 
past 4 weeks. Psychotropic medication use in the past 4 weeks was assessed on each follow-up questionnaire. Psychotropic medications were defined as anxiolytics, anticonvulsants, antipsychotics, atypical antidepressants, benzodiazepines, beta-blockers, mood stabilizers, sedative-hypnotics, selective serotonin-norepinephrine reuptake inhibitors, selective serotonin reuptake inhibitors, stimulants, tetracyclic antidepressants, and tricyclic antidepressants. Psychotropic medication was categorized as never, former, and current use.

\section{Assessment of covariates}

Covariates were ascertained at baseline via self-report and included factors that have been associated with depression or menstrual cycle characteristics in previous research..$^{25-27}$ These included age, race/ethnicity, education, income, height, weight, physical activity, parity, smoking, alcohol and caffeine intake, and last method of contraception. Body mass index (BMI) was calculated as weight $(\mathrm{kg}) /$ height $\left(\mathrm{m}^{2}\right)$. Total metabolic equivalents (METs) of physical activity were calculated by multiplying the average number of hours per week engaging in various activities by metabolic equivalents estimated from the Compendium of Physical Activities. ${ }^{28,29}$

\section{Exclusions}

For this analysis, 6,867 women who enrolled from June 2013 through January 2018 completed the baseline questionnaire. We excluded women with reported last menstrual period (LMP) date at least 6 months before baseline $(n=87)$, with baseline LMP dates later than the date of the baseline questionnaire $(n=11)$, and who were pregnant at baseline $(n=54)$. We also excluded women who had been on hormones most of the time and were unable to report their cycle regularity off hormones on the baseline questionnaire $(n=1,276)$; these women were less likely to have had a pregnancy in the past (16.7\% vs $34.3 \%)$, reported lower baseline depressive symptoms (mean MDI score 9.6 vs 12.0) and perceived stress (mean PSS-10 score 15.7 vs 16.7), and were more likely to be a current psychotropic medication user $(13.9 \%$ vs $11.7 \%$ ) when compared with women who were included in the final sample.

For our primary analysis of mental health and menstrual cycle characteristics (ie, cycle regularity and cycle length), we additionally excluded women who did not complete any follow-up questionnaires. Of the women who did not complete any follow-up questionnaires $(n=2,093), 1,336$ were lost to follow-up (65\%); 412 women reported a miscarriage, pregnancy, or were found in birth/baby registries (20\%); 287 were not pregnant at study withdrawal (14\%); 32 reported no longer trying to conceive $(2 \%)$; and 6 reported fertility treatment $(<1 \%)$, leaving a final sample of 3,346 . For the secondary cross-sectional analysis of mental health and baseline menstrual cycle characteristics (ie, bleed length and heaviness of flow), which did not require any follow-up data, the study population was 5,439.

\section{Data analysis}

Our primary analysis assessed the relationship between depressive symptoms, perceived stress, and psychotropic medication use and subsequent cycle regularity and length. We used the Anderson-Gill data structure to allow for one observation per woman per follow-up cycle. Women contributed up to six separate observations to the analysis, equal to the number of follow-up questionnaires they completed (median $=2$ follow-up questionnaires, range $=1-6$ ). Study outcomes (irregular cycles [yes vs no] and cycle length [categorized as $<25,25-34$, and $\geq 35$ days]) were measured during each 2-month follow-up period, based on what was reported in the follow-up questionnaires. The effect of time-varying exposures measured at baseline and follow-up (ie, PSS-10 score and psychotropic medication use) was evaluated only in relation to outcomes on the subsequent questionnaire, approximately 2 months later. For example, for PSS-10 ascertained on the baseline questionnaire, menstrual characteristics were measured on the first follow-up questionnaire; for PSS-10 ascertained on the first follow-up questionnaire, menstrual characteristics were measured on the second follow-up questionnaire, and so on. Exposures measured at baseline only (ie, MDI score) were considered constant during the follow-up period.

We used log-binomial regression models to estimate prevalence ratios (PR) and 95\% confidence intervals (CI), adjusting for the following covariates: age $(<25,25-29$, $30-34, \geq 35$ years), race/ethnicity (Non-Hispanic white: yes vs no), annual household income $(<\$ 50,000, \$ 50,000-\$ 99,999$, $\$ 100,000-\$ 149,999, \geq \$ 150,000$ USD), education (less than college degree, college degree, graduate school), BMI $(<25$, $\left.25-29,30-34, \geq 35 \mathrm{~kg} / \mathrm{m}^{2}\right)$, physical activity $(<10,10-19$, 20-39, $\geq 40$ MET-hours/week), cigarette smoking (current regular, current occasional, former, never), alcohol use ( 0 , $1-6,7-13, \geq 14$ drinks/week), caffeine intake (<100, 100-199, 200-299, $\geq 300 \mathrm{mg} /$ day), parity (nulliparous, parous), and last method of contraception (hormonal, barrier, withdrawal/ rhythm methods). We used generalized estimating equations with an exchangeable correlation matrix to account for correlation between multiple observations per woman. ${ }^{30}$ In order to assess the possibility of reverse causation (ie, irregular 
cycles predicting mental health symptoms as opposed to mental health symptoms predicting irregular cycles), we conducted a sensitivity analysis restricted to women who did not report irregular cycles at baseline $(n=2,409)$ and included their follow-up intervals until the report of irregular cycles. In this analysis, we estimated risk ratios (RR) and 95\% CI, adjusting for all of the same covariates.

In secondary cross-sectional analyses based on the baseline data only, we assessed the association of mental health with bleed length and heaviness of bleed. We used log-binomial regression models to estimate PRs and 95\% CIs, adjusting for the covariates listed above.

Missingness in baseline covariates ranged from $0 \%$ (age, race/ethnicity, parity) to $4.7 \%$ (income). For time-varying exposure (ie, PSS-10 and psychotropic medication use) and outcome (ie, cycle regularity and length) variables, missing observations were carried forward by using the most recent observed time point. We employed multiple imputation to address missing baseline data on exposures and covariates using PROC MI in SAS (SAS Institute version 9.3). We generated five imputed data sets and combined point estimates and standard errors using PROC MIANALYZE.

Our cohort comprised pregnancy planners who had been attempting pregnancy for varying lengths of time at study entry. If women with suboptimal menstrual cycle characteristics took longer to conceive, and this contributed to declines in mental health, our analysis could be affected by reverse causation. To assess this possibility, we repeated all analyses after restricting to women who had been attempting to conceive for $<3$ cycles at study entry.

\section{Results}

Mean depressive symptom score at baseline was 10.6 (SD $=8.5$ ) and ranged from 0 to 50 with $4.4 \%$ of women classified as having severe depression. Mean perceived stress score at baseline was $16.0(\mathrm{SD}=5.8)$ and ranged from 0 to 36 with $8.5 \%$ of women with PSS- 10 scores $\geq 25$. A total of $32.5 \%$ of women reported a history of depression or anxiety $(11.0 \%$ reported both). Overall, $16.1 \%$ and $11.2 \%$ of women were former or current users of psychotropic medication, respectively. A total of 500 (out of 2,409 ) regularly cycling women at baseline (24.4\%) reported irregular cycles over the course of the follow-up intervals.

Women with severe depressive symptoms were more likely to smoke and had higher caffeine intake than women with no or mild depressive symptoms. Similarly, women with higher levels of perceived stress were more likely to smoke and had higher caffeine intake than women with lower levels of perceived stress. Women with severe depressive symptoms were less likely to have a college degree and were more likely to have an annual income of $<\$ 50,000$ than women with no or mild depressive symptoms. The same pattern emerged for women who reported higher levels of perceived stress vs lower levels of perceived stress. Differences in baseline characteristics among never, former, and current users of psychotropic medication were less pronounced, but trended in the same direction. Depressive symptoms and perceived stress were positively correlated $(r=0.65)$. In addition, current or former users of psychotropic medication had more depressive symptoms and perceived stress at baseline. Additional characteristics of the sample can be found in Table 1.

\section{Mental health, psychotropic medication use, and irregular cycles}

Women with severe depressive symptoms at baseline had a $63 \%(95 \% \mathrm{CI}=1.36-1.95$; Table 2$)$ greater prevalence of irregular cycles during follow-up compared with women with no/low depressive symptoms. Likewise, higher perceived stress was associated with the prevalence of irregular cycles during follow-up (PR for a score of $\geq 25$ vs $<15=1.31,95 \%$ $\mathrm{CI}=1.15-1.48)$. Results were similar with and without adjustment for demographic factors. Analyses restricted to women who reported regular cycles at baseline revealed similar results (Table 2).

Current psychotropic medication use was not associated with prevalence of irregular cycles during follow-up compared with never users $(\mathrm{PR}=0.90,95 \% \mathrm{CI}=0.73,1.10)$, while former psychotropic medication use was associated with slightly lower prevalence of irregular cycles during follow-up compared with never users ( $\mathrm{PR}=0.83,95 \% \mathrm{CI}$ $=0.68,1.02)$. However, among regularly cycling women at baseline, former $(\mathrm{RR}=0.97,95 \% \mathrm{CI}=0.68,1.39)$ and current $(\mathrm{RR}=1.15,95 \% \mathrm{CI}=0.80,1.68)$ psychotropic medication use were not strongly associated with a greater risk of irregular cycles when compared with never use of psychotropic medication (Table 2).

Examination of depressive symptoms in conjunction with medication use revealed that more severe depressive symptoms were associated with a higher prevalence of irregular cycles regardless of psychotropic medication use history (Table 2). Similarly, greater perceived stress was associated with a higher prevalence of irregular cycles regardless of psychotropic medication use. Analyses conducted among regularly cycling women only at baseline also suggested a greater risk of irregular cycles among women who reported severe depressive symptoms and higher perceived stress, 
Table I Baseline characteristics ${ }^{\mathrm{a}}$ of 3,346 women by MDI score, PSS-I0 score, and psychotropic medication use, PRESTO, 20I3-20I8

\begin{tabular}{|c|c|c|c|c|c|c|c|c|c|c|c|}
\hline \multirow[t]{2}{*}{ Characteristic } & \multicolumn{4}{|c|}{ MDI score } & \multicolumn{4}{|c|}{ PSS-10 score } & \multicolumn{3}{|c|}{$\begin{array}{l}\text { Psychotropic } \\
\text { medication use }\end{array}$} \\
\hline & $<20$ & $20-24$ & $25-29$ & $\geq 30$ & $<15$ & $15-19$ & $20-24$ & $\geq 25$ & Never & Former & Current \\
\hline \multirow[t]{2}{*}{ Number of women (\%) } & 2,862 & 213 & 124 & 147 & 1,425 & 1,058 & 579 & 284 & 2,431 & 540 & 375 \\
\hline & $(85.5)$ & $(6.4)$ & $(3.7)$ & $(4.4)$ & $(42.6)$ & $(31.6)$ & $(17.3)$ & $(8.5)$ & $(72.7)$ & $(16.1)$ & $(11.2)$ \\
\hline Age, years (mean) & 30.6 & 29.7 & 29.2 & 29.0 & 30.7 & 30.4 & 30.2 & 29.5 & 30.4 & 30.4 & 30.7 \\
\hline Non-Hispanic White (\%) & 83.7 & 80.2 & 78.9 & 78.6 & 83.6 & 83.6 & 81.7 & 80.5 & 82.1 & 84.4 & 87.3 \\
\hline Less than college degree (\%) & 21.6 & 36.4 & 40.9 & 52.1 & 18.2 & 25.0 & 30.5 & 40.7 & 22.1 & 31.0 & 31.2 \\
\hline Annual household income $<\$ 50,000(\%)$ & 16.7 & 28.8 & 35.6 & 44.0 & 14.3 & 19.3 & 24.6 & 35.6 & 18.3 & 21.3 & 24.0 \\
\hline BMl, kg/m² (mean) & 27.1 & 30.0 & 30.3 & 31.4 & 26.6 & 27.6 & 28.7 & 29.7 & 27.2 & 27.7 & 29.9 \\
\hline Physical activity, MET-hours/week (mean) & 35,6 & 33.4 & 29.7 & 27.9 & 37.4 & 34.1 & 33.4 & 28.2 & 35.4 & 34.6 & 32.1 \\
\hline Current regular smoker (\%) & 4.7 & 8.4 & 11.2 & 16.5 & 4.0 & 6.3 & 7.4 & 8.2 & 5.1 & 6,4 & 8.6 \\
\hline Current alcohol intake, drinks/week (mean) & 3.1 & 3.3 & 3.5 & 3.8 & 3.2 & 3.3 & 2.9 & 3.6 & 3.1 & 3.5 & 3.3 \\
\hline Current caffeine intake, mg/day (mean) & 115.8 & 120.1 & 129.3 & 163.7 & 112.3 & 123.6 & 114.5 & 141.0 & 112.2 & 127.5 & 146.8 \\
\hline Parous (\%) & 31.7 & 38.8 & 33.6 & 34.2 & 30.8 & 31.3 & 36.7 & 34.8 & 32.4 & 32.9 & 31.8 \\
\hline Last method of contraception - hormonal (\%) & 29.8 & 32.6 & 31.5 & 34.6 & 29.5 & 29.9 & 30.5 & 34.9 & 30.0 & 28.8 & 35.3 \\
\hline Age at menarche, years (mean) & 12.4 & 12.3 & 12.5 & 12.2 & 12.5 & 12.4 & 12.3 & 12.4 & 12.4 & 12.4 & 12.4 \\
\hline Irregular cycles (\%) & 21.1 & 28.9 & 32.1 & 46.1 & 19.6 & 23.8 & 23.8 & 35.8 & 22.6 & 24.9 & 24.2 \\
\hline Cycle length, days (mean) & 28.8 & 28.5 & 29.2 & 29.0 & 28.9 & 28.8 & 28.7 & 28.6 & 28.8 & 28.8 & 28.7 \\
\hline Bleed length $\geq 5$ days (\%) & 45.7 & 52.7 & 46.6 & 47.7 & 44.6 & 45.9 & 47.3 & 52.7 & 46.2 & 47.7 & 45.2 \\
\hline Heavy bleeds (\%) & 25.0 & 39.9 & 30.4 & 38.5 & 23.5 & 25.1 & 30.3 & 39.4 & 25.8 & 28.4 & 30.2 \\
\hline Current psychotropic medication use (\%) & 9.5 & 17.4 & 20.7 & 29.3 & 7.1 & 10.0 & 16.5 & 25.1 & - & - & - \\
\hline MDI score (mean) & - & - & - & - & 6.1 & 10.3 & 15.3 & 24.4 & 9.4 & 13.1 & 14.6 \\
\hline PSS-10 score (mean) & 14.8 & 21.2 & 22.7 & 25.7 & - & - & - & - & 15.1 & 17.8 & 18.6 \\
\hline History of depression or anxiety & 28.6 & 47.6 & 54.5 & 72.1 & 21.7 & 32.6 & 45.3 & 59.8 & 8.2 & 100.0 & 93.1 \\
\hline
\end{tabular}

Notes: aWith the exception of age, all characteristics are age-standardized to the age distribution of the study population at baseline.

Abbreviations: BMI, body mass index; MDI, Major Depression Inventory; MET, metabolic equivalent of task; PRESTO, Pregnancy Study Online; PSS, Perceived Stress Scale.

controlling for psychotropic medication use. These analyses also showed that women with severe depressive symptoms and high perceived stress, who also currently use psychotropic medications, had the highest risk of irregular cycles (Table 2).

\section{Mental health, psychotropic medication use, and menstrual cycle length}

There were few consistent associations between the selected mental health variables and prospectively assessed menstrual cycle length (Table 3). Severe depressive symptoms (MDI score $\geq 30$ ) and higher levels of perceived stress were associated with a higher prevalence of having short cycle lengths. However, the estimates were imprecise and numbers of women with short ( $<25$ days) and long ( $\geq 35$ days) cycles were small.

\section{Mental health, psychotropic medication use, and heavy bleeds}

In cross-sectional analyses, higher levels of perceived stress were associated with a slightly higher prevalence of heavy bleeds after adjustment for demographic factors and medication use. Moderate, but not severe levels, of baseline depressive symptoms were associated with a slightly higher prevalence of heavy bleeds (Table 4). There was little association between psychotropic medication use and prevalence of heavy bleeds.

Examination of depressive symptoms in conjunction with medication use revealed that severe depressive symptoms with concurrent psychotropic medication use at baseline was modestly associated with a greater prevalence of heavy bleeds ( $\mathrm{PR}=1.18,95 \% \mathrm{CI}=0.96-1.44)$ relative to low depressive symptoms without psychotropic medication use. Higher perceived stress was associated with a greater prevalence of heavy bleeds regardless of psychotropic medication use (Table 4).

\section{Mental health, psychotropic medication use, and bleed length}

We found little association between any of the mental health exposures and the prevalence of short or long bleeds (Table S1).

All results were similar among women who had been attempting to conceive for $<3$ cycles at study entry (data not shown).

\section{Discussion}

In this prospective cohort study of women attempting to conceive, depressive symptoms and perceived stress were 
Table 2 Depression, stress, anxiety, and psychotropic medication use in relation to prevalence of prospectively assessed cycle irregularity among 3,346 pregnancy planners, PRESTO, 2013-2018

\begin{tabular}{|c|c|c|c|c|c|}
\hline & \multicolumn{3}{|l|}{ All women } & \multicolumn{2}{|c|}{$\begin{array}{l}\text { Regularly cycling women } \\
\text { at baseline }{ }^{\text {a }}\end{array}$} \\
\hline & $\begin{array}{l}\text { Irregular } \\
\text { cycles/Total }\end{array}$ & $\begin{array}{l}\text { Unadjusted } \\
\text { PR (95\% CI) }\end{array}$ & $\begin{array}{l}\text { Adjusted }^{\mathrm{b}} \text { PR } \\
(95 \% \mathrm{Cl})\end{array}$ & $\begin{array}{l}\text { Irregular } \\
\text { cycles/Total }^{\mathrm{a}}\end{array}$ & $\begin{array}{l}\text { Adjusted }{ }^{a, b} \\
\text { RR }(95 \% \mathrm{Cl})\end{array}$ \\
\hline \multicolumn{6}{|l|}{ MDI score ${ }^{c}$} \\
\hline$<20$ & $1,430 / 6,829$ & Reference & Reference & $425 / 4,713$ & Reference \\
\hline $20-24$ & $167 / 555$ & $1.33(1.08-1.62)$ & $1.19(0.97-1.44)$ & $33 / 321$ & $1.09(0.78-1.54)$ \\
\hline $25-29$ & $87 / 324$ & $1.28(0.98-1.66)$ & $1.13(0.88-1.46)$ & $18 / 202$ & $0.89(0.55-1.44)$ \\
\hline$\geq 30$ & $|4| / 33 \mid$ & $2.01(1.68-2.40)$ & $1.63(1.36-1.95)$ & $24 / 133$ & $1.75(1.22-2.52)$ \\
\hline \multicolumn{6}{|l|}{ PSS- 10 score } \\
\hline$<15$ & $705 / 3,493$ & Reference & Reference & $217 / 2,427$ & Reference \\
\hline $15-19$ & $484 / 2,343$ & $1.06(0.96-1.16)$ & $1.03(0.94-1.13)$ & $129 / 1,625$ & $0.87(0.7 I-I .07)$ \\
\hline $20-24$ & $387 / 1,428$ & $1.23(1.10-1.38)$ & $1.17(1.05-1.31)$ & $101 / 897$ & $1.20(0.96-1.50)$ \\
\hline$\geq 25$ & $249 / 775$ & $1.43(1.25-1.64)$ & $1.31(1.15-1.48)$ & $53 / 420$ & $1.33(1.00-1.76)$ \\
\hline \multicolumn{6}{|c|}{ Psychotropic medication use } \\
\hline Never & $1,286 / 5,804$ & Reference & Reference & $351 / 3,936$ & Reference \\
\hline Former & $317 / 1,395$ & $1.04(0.90-1.19)$ & $0.83(0.68-1.02)$ & $84 / 888$ & $0.97(0.68-1.39)$ \\
\hline Current & $222 / 840$ & I.I $3(0.97-1.32)$ & $0.90(0.73-1.10)$ & $65 / 545$ & I.I $5(0.80-1.68)$ \\
\hline \multicolumn{6}{|c|}{ MDI score and medication history ${ }^{c}$} \\
\hline $\mathrm{MDI}<25$, never treated & $1,183 / 5,506$ & Reference & Reference & $332 / 3,783$ & Reference \\
\hline $\mathrm{MDI}<25$, formerly treated & $242 / 1,174$ & $0.98(0.84-1.14)$ & $0.95(0.8 I-I . I I)$ & $71 / 776$ & $1.07(0.83-1.37)$ \\
\hline $\mathrm{MDI}<25$, currently treated & I72/704 & $1.06(0.89-1.26)$ & $1.00(0.85-1.18)$ & $55 / 475$ & $1.30(0.99-1.7 I)$ \\
\hline $\mathrm{MDI} \geq 25$, never treated & $103 / 298$ & $1.59(1.27-1.99)$ & $1.36(1.11-1.67)$ & $19 / 153$ & $1.28(0.8 I-2.0 \mathrm{I})$ \\
\hline $\mathrm{MDI} \geq 25$, formerly treated & $75 / 221$ & $1.60(1.25-2.06)$ & $1.35(1.05-1.73)$ & $13 / 112$ & $1.20(0.69-2.10)$ \\
\hline MDI $\geq 25$, currently treated & $50 / 136$ & $1.74(1.33-2.28)$ & $1.40(1.07-1.83)$ & $10 / 70$ & $\mathrm{I} .45(0.8 \mathrm{I}-2.59)$ \\
\hline \multicolumn{6}{|c|}{ PSS-10 score and medication history } \\
\hline PSS $<20$, never treated & $916 / 4,519$ & Reference & Reference & $27 \mid / 3,171$ & Reference \\
\hline PSS $<20$, formerly treated & $165 / 857$ & $0.94(0.79-1.12)$ & $0.92(0.77-1.08)$ & $45 / 563$ & $0.96(0.7 I-I .30)$ \\
\hline PSS $<20$, currently treated & $108 / 460$ & $1.12(0.93-1.36)$ & I.06 (0.88-I.27) & $30 / 318$ & $1.09(0.75-1.58)$ \\
\hline PSS $\geq 20$, never treated & $370 / 1,285$ & $1.22(1.09-1.37)$ & $1.18(1.06-1.32)$ & $80 / 765$ & $1.18(0.94-1.50)$ \\
\hline PSS $\geq 20$, formerly treated & $152 / 538$ & $1.32(1.12-1.56)$ & $1.20(1.02-1.42)$ & $39 / 325$ & $1.37(0.99,1.89)$ \\
\hline PSS $\geq 20$, currently treated & $114 / 380$ & $1.29(1.08-1.56)$ & $1.15(0.96-1.38)$ & $35 / 227$ & $1.69(1.23,2.32)$ \\
\hline
\end{tabular}

Notes: ${ }^{a}$ Analysis excludes women with irregular cycles at baseline and includes cycles of observation until occurrence of irregular cycles or end of follow-up (ie, first occurrence of irregular cycles only). bAdjusted for age, race/ethnicity, income, education, BMI, physical activity, smoking history, alcohol use, parity, last method of contraception, and history of anxiety and/or depression diagnosis (model for psychotropic medication use only). 'MDI score measured at baseline only.

Abbreviations: BMI, body mass index; MDI, Major Depression Inventory; PR, prevalence ratios, PRESTO, Pregnancy Study Online; PSS, Perceived Stress Scale; RR, risk ratio.

positively associated with cycle irregularity. Specifically, women who reported higher levels of depressive symptoms and perceived stress had a greater prevalence $(63 \%$ and $31 \%$, respectively) of irregular cycles over follow-up. The association persisted among regularly cycling women at baseline than women with lower levels of depressive symptoms and perceived stress, respectively. Current psychotropic medication use was not associated with a greater prevalence of irregular cycles. There were few consistent associations between mental health and psychotropic medication use and cycle length. Similar patterns of results were found in cross-sectional analyses examining the prevalence of heavy bleeds at baseline. Specifically, baseline depressive symptoms and perceived stress were associated with heavier bleeds, regardless of psychotropic medication use. In general, mental health and psychotropic medication use showed little association with bleed length.
Among the study population as a whole, higher levels of depressive symptoms at baseline and elevated levels of perceived stress over follow-up were associated with a greater prevalence of cycle irregularity, and psychotropic medication use (never, former, or current) did not appear to explain this association. These findings are consistent with the idea that depressive symptoms and perceived stress, as opposed to their pharmacological treatment, may be more important predictors of cycle irregularity.

Results of this study align with previous retrospective ${ }^{3,12,13}$ and prospective ${ }^{14}$ studies, which have documented the association of depression and perceived stress with menstrual cycle characteristics. The current study extends the literature by examining the association of depressive symptoms, perceived stress, and psychotropic medication use individually and jointly on prospectively assessed 
Table 3 Depression, stress, anxiety, and psychotropic medication use in relation to prevalence of prospectively assessed cycle length among 2,409 regularly cycling (at baseline) pregnancy planners, PRESTO, 2013-2018

\begin{tabular}{|c|c|c|c|c|c|c|}
\hline & \multicolumn{3}{|c|}{ Cycle length $<25$ days $^{a}$} & \multicolumn{3}{|c|}{ Cycle length $\geq 35$ days $^{a}$} \\
\hline & Event/ Total & $\begin{array}{l}\text { Unadjusted PR } \\
(95 \% \mathrm{Cl})\end{array}$ & $\begin{array}{l}\text { Adjusted }^{\mathrm{b}} \text { PR } \\
(95 \% \mathrm{Cl})\end{array}$ & Event/ Total & $\begin{array}{l}\text { Unadjusted PR } \\
(95 \% \mathrm{Cl})\end{array}$ & $\begin{array}{l}\text { Adjusted }^{\mathrm{b}} \text { PR } \\
(95 \% \mathrm{Cl})\end{array}$ \\
\hline \multicolumn{7}{|l|}{ MDI score ${ }^{c}$} \\
\hline$<20$ & $193 / 5,380$ & Reference & Reference & $380 / 5,380$ & Reference & Reference \\
\hline $20-24$ & $16 / 384$ & $1.19(0.64-2.19)$ & $1.14(0.63-2.08)$ & $25 / 384$ & $1.06(0.65-1.71)$ & $0.95(0.60-1.52)$ \\
\hline $25-29$ & $5 / 235$ & $0.70(0.28-1.74)$ & $0.65(0.26-1.62)$ & $22 / 235$ & $1.40(0.87-2.26)$ & $1.20(0.74-1.96)$ \\
\hline$\geq 30$ & $10 / 187$ & I.7I (0.88-3.32) & $1.50(0.77-2.93)$ & $12 / 187$ & $0.90(0.46-1.75)$ & $0.79(0.42-1.49)$ \\
\hline \multicolumn{7}{|l|}{ PSS-I 0 score } \\
\hline$<15$ & $84 / 2,778$ & Reference & Reference & $192 / 2,778$ & Reference & Reference \\
\hline $15-19$ & $70 / 1,848$ & $1.31(0.96-1.77)$ & $1.28(0.94-1.74)$ & $129 / 1,848$ & $1.10(0.91-1.34)$ & $1.05(0.86-1.29)$ \\
\hline $20-24$ & $46 / 1,037$ & $1.42(0.97-2.08)$ & $1.41(0.96-2.05)$ & $78 / 1,037$ & $1.18(0.93-1.49)$ & I. $10(0.87-1.40)$ \\
\hline$\geq 25$ & $24 / 523$ & $1.48(0.93-2.35)$ & $1.44(0.90-2.29)$ & $40 / 523$ & $1.17(0.85-1.60)$ & $1.07(0.78-1.48)$ \\
\hline \multicolumn{7}{|c|}{ Psychotropic medication use } \\
\hline Never & $170 / 4,503$ & Reference & Reference & $300 / 4,503$ & Reference & Reference \\
\hline Former & $29 / 1,070$ & $0.69(0.44-1.07)$ & $0.61(0.30-1.25)$ & $92 / 1,070$ & $1.22(0.94-1.59)$ & $1.19(0.75-1.89)$ \\
\hline Current & $25 / 613$ & $\mathrm{I} .07(0.67-\mathrm{I} .72)$ & $0.94(0.44-1.99)$ & $47 / 613$ & I.II (0.8I-I.53) & $1.02(0.63-1.67)$ \\
\hline \multicolumn{7}{|c|}{ MDI and medication history ${ }^{c}$} \\
\hline MDI $<25$, never treated & $162 / 4,310$ & Reference & Reference & $285 / 4,310$ & Reference & Reference \\
\hline MDI $<25$, formerly treated & $26 / 926$ & $0.69(0.43-\mathrm{I} .12)$ & $0.66(0.4 I-1.06)$ & $77 / 926$ & $1.20(0.90-1.59)$ & $1.14(0.86-1.52)$ \\
\hline MDI $<25$, currently treated & $21 / 528$ & $1.04(0.63-1.73)$ & $0.99(0.59-1.65)$ & $43 / 528$ & $1.22(0.87-1.70)$ & $1.14(0.8 \mathrm{I}-\mathrm{I} .58)$ \\
\hline $\mathrm{MDI} \geq 25$, never treated & $8 / 193$ & $1.23(0.59-2.61)$ & $\mathrm{I} .14(0.54-2.4 \mathrm{I})$ & $15 / 193$ & $1.29(0.75-2.23)$ & $1.10(0.64-1.91)$ \\
\hline MDI $\geq 25$, formerly treated & $3 / 144$ & $0.73(0.26-2.04)$ & $0.65(0.23-1.88)$ & $15 / 144$ & $1.53(0.87-2.71)$ & $1.48(0.85-2.58)$ \\
\hline MDI $\geq 25$, currently treated & $4 / 85$ & $1.30(0.39-4.36)$ & $1.13(0.34-3.70)$ & $4 / 85$ & $0.63(0.26-1.49)$ & $0.51(0.22-1.19)$ \\
\hline \multicolumn{7}{|c|}{ PSS- 10 and medication history } \\
\hline PSS $<20$, never treated & $124 / 3,590$ & Reference & Reference & $234 / 3,590$ & Reference & Reference \\
\hline PSS $<20$, formerly treated & $16 / 686$ & $0.67(0.40-1.13)$ & $0.63(0.38-1.06)$ & $61 / 686$ & $1.21(0.89-1.63)$ & $1.16(0.85-1.57)$ \\
\hline PSS $<20$, currently treated & $14 / 350$ & $1.13(0.65-1.96)$ & $1.07(0.6 \mathrm{I}-1.87)$ & $26 / 350$ & $1.08(0.72-1.62)$ & $1.00(0.67-1.50)$ \\
\hline PSS $\geq 20$, never treated & $46 / 913$ & $1.35(0.94-1.94)$ & $1.34(0.94-1.92)$ & $66 / 913$ & $1.09(0.86-1.39)$ & $1.04(0.82-1.33)$ \\
\hline PSS $\geq 20$, formerly treated & $13 / 384$ & $0.85(0.44-1.64)$ & $0.82(0.42-1.58)$ & $31 / 384$ & $1.33(0.93-1.90)$ & $1.25(0.87-1.80)$ \\
\hline PSS $\geq 20$, currently treated & $11 / 263$ & $1.19(0.60-2.36)$ & $1.10(0.55-2.20)$ & $21 / 263$ & $1.20(0.80-1.82)$ & $1.07(0.70-1.63)$ \\
\hline
\end{tabular}

Notes: a Compared to reference group of cycle length 25-34 days. ${ }^{b}$ Adjusted for age, race/ethnicity, income, education, BMI, physical activity, smoking history, parity, last method of contraception, and history of anxiety and/or depression diagnosis (model for psychotropic medication use only). ${ }^{\mathrm{C}} \mathrm{MDI}$ score measured at baseline only.

Abbreviations: BMI, body mass index; MDI, Major Depression Inventory; PR, prevalence ratios; PRESTO, Pregnancy Study Online; PSS, Perceived Stress Scale.

cycle irregularity. Prospective follow-up in relation to current measurement of perceived stress is an important strength of this study, given that repeated assessments of perceived stress are more predictive of ovulatory function than baseline level of perceived stress. ${ }^{14}$ However, results diverge from previous work demonstrating an association between psychotropic medication and polycystic ovary syndrome, a condition defined by menstrual cycle irregularity. ${ }^{15,16}$ Specifically, previous work has found that valproate, an antiepileptic drug that is used to treat mood disorders (ie, bipolar disorder), is associated with polycystic ovary syndrome. ${ }^{15,16}$ Although the current study captured valproate use and also examined a mood disorder, the current study differed from these previous studies in that all types of psychotropic medications were combined into a single variable. The small numbers across the different classes of psychotropic medications, including valproate, limited our ability to examine the effects of individual medications on menstrual cycle characteristics.

A potential mechanism linking depression and perceived stress with menstrual cycle irregularity is the influence of the HPA axis on gonadotropin-releasing hormone (GnRH). Depression and perceived stress are associated with dysregulation of the HPA axis..$^{8-10}$ In turn, HPA axis dysregulation has an inhibitory effect on $\mathrm{GnRH}$, which subsequently delays or impedes the LH surge. ${ }^{11,31}$ In fact, animal models have shown that administration of cortisol - a steroid hormone released in response to stress - delays the rise in estradiol in the follicular phase and interferes with the LH and folliclestimulating hormone surges that occur prior to ovulation. ${ }^{11}$

There are several study limitations worth noting. First, although we followed women prospectively, we were unable to assess the precise onset of menstrual cycle irregularity in relation to mental health symptoms. Therefore, we cannot 
Table 4 Cross-sectional analysis of depression, stress, anxiety, and psychotropic medication use in relation to prevalence of heavy bleeds, PRESTO, 2013-2018

\begin{tabular}{|c|c|c|c|}
\hline & $\begin{array}{l}\text { Heavy bleeds/ } \\
\text { Total }\end{array}$ & $\begin{array}{l}\text { Unadjusted PR } \\
(95 \% \mathrm{Cl})\end{array}$ & $\begin{array}{l}\text { Adjusted }^{a} \text { PR } \\
(95 \% \mathrm{Cl})\end{array}$ \\
\hline \multicolumn{4}{|l|}{ MDI score } \\
\hline$<20$ & $1,153 / 4,388$ & Reference & Reference \\
\hline $20-24$ & $15|/ 4| 0$ & $1.40(1.22-1.60)$ & $1.21(1.06-1.39)$ \\
\hline $25-29$ & $91 / 272$ & $1.27(1.07-1.52)$ & $1.13(0.95-1.34)$ \\
\hline$\geq 30$ & $146 / 396$ & $1.41(1.23-1.62)$ & $1.10(0.96-1.28)$ \\
\hline \multicolumn{4}{|l|}{ PSS-I 0 score } \\
\hline$<15$ & $509 / 2,073$ & Reference & Reference \\
\hline $15-19$ & $468 / 1,703$ & $1.12(1.00-1.25)$ & $1.07(0.96-1.19)$ \\
\hline $20-24$ & $338 / 1,097$ & $1.25(I . \mid 2-1.4 I)$ & I.II (0.99-I.25) \\
\hline$\geq 25$ & $226 / 593$ & $1.55(1.37-1.77)$ & $1.29(1.13-1.47)$ \\
\hline \multicolumn{4}{|c|}{ Psychotropic medication use } \\
\hline Never & $1,079 / 3,962$ & Reference & Reference \\
\hline Former & $262 / 864$ & $1.11(1.00-1.25)$ & $0.94(0.79-1.10)$ \\
\hline Current & $200 / 640$ & $1.15(1.01-1.30)$ & $0.91(0.77-1.08)$ \\
\hline \multicolumn{4}{|c|}{ MDI score and medication history } \\
\hline $\mathrm{MDI}<25$, never treated & $957 / 3,613$ & Reference & Reference \\
\hline $\mathrm{MDI}<25$, formerly treated & $209 / 708$ & $1.12(0.98-1.27)$ & $1.10(0.97-1.24)$ \\
\hline $\mathrm{MDI}<25$, currently treated & | 38/477 & $1.09(0.94-1.27)$ & $1.02(0.87-1.18)$ \\
\hline$M D I \geq 25$, never treated & $122 / 349$ & $1.33(1.14-1.54)$ & $1.09(0.94-1.28)$ \\
\hline MDI $\geq 25$, formerly treated & $53 / 156$ & $1.28(1.03-1.61)$ & $1.02(0.82-1.28)$ \\
\hline MDI $\geq 25$, currently treated & $62 / 163$ & $1.44(1.17-1.76)$ & $1.18(0.96-1.44)$ \\
\hline \multicolumn{4}{|c|}{ PSS-I 0 score and medication history } \\
\hline PSS $<20$, never treated & $759 / 2,970$ & Reference & Reference \\
\hline PSS $<20$, formerly treated & $131 / 489$ & $1.05(0.89-1.23)$ & $1.03(0.88-1.21)$ \\
\hline PSS $<20$, currently treated & $87 / 317$ & $1.08(0.89-1.30)$ & $1.01(0.84-1.22)$ \\
\hline PSS $\geq 20$, never treated & $320 / 992$ & $1.26(I . \mid 3-1.4 I)$ & $1.12(1.01-1.25)$ \\
\hline PSS $\geq 20$, formerly treated & $13 \mid / 375$ & $1.37(1.18-1.59)$ & $1.18(1.02-1.37)$ \\
\hline PSS $\geq 20$, currently treated & $113 / 323$ & $1.36(1.16-1.60)$ & $1.16(0.98-1.36)$ \\
\hline
\end{tabular}

Notes: adjusted for age, race/ethnicity, income, education, BMI, physical activity, smoking history, alcohol use, caffeine intake, parity, last method of contraception, and history of anxiety and/or depression diagnosis (models for psychotropic medication use only).

Abbreviations: BMI, body mass index; MDI, Major Depression Inventory; PR, prevalence ratios; PRESTO, Pregnancy Study Online; PSS, Perceived Stress Scale.

determine whether menstrual cycle disturbances (eg, cycle irregularity) influenced a change in mood, or vice versa. To address this limitation, we conducted sensitivity analyses in which we excluded women with irregular cycles at baseline and found largely similar results. Second, self-reported depressive symptoms were assessed only at baseline. Consequently, we were unable to examine the effect of time-varying depressive symptoms on menstrual cycle characteristics during follow-up as we did for perceived stress and psychotropic medication use. Third, we did not ask about nonpharmacologic types of treatment for depression such as cognitive behavioral therapy, which could ameliorate psychological symptoms. ${ }^{32}$ Fourth, our follow-up period was short (ie, 12 months at most), so we were unable to look at the longer term effects of depressive symptoms and perceived stress on cycle irregularity. Fifth, heaviness of flow was assessed via number of tampons or pads used per menses. Given that women may change their tampons or pads at different frequencies for a variety of reasons other than bleeding, this may not always accurately reflect heaviness of flow. Finally, only $8.1 \%$ of the study population reported moderate to severe depressive symptoms, indicating that most women were not experiencing depressive symptoms at baseline. However, the prevalence of moderate to severe depressive symptoms in the study population is similar to the prevalence of depression (6.6\%) during a 12-month period in the USA population. ${ }^{27}$

In summary, we found that depressive symptoms and perceived stress were associated with an increased prevalence of cycle irregularity over time. Psychotropic medication use showed little association with cycle irregularity in the entire cohort. These results indicate that mental health may be an important determinant of cycle regularity. Interventions aimed at improving mental health, particularly during the reproductive years, may be important for fertility. 


\section{Acknowledgments}

This research was supported by NICHD grants (K23HD087428, R21-HD072326, R01-HD086742, T32HD052458).

\section{Disclosure}

The authors report no conflicts of interest in this work.

\section{References}

1. Solomon CG, Hu FB, Dunaif A, et al. Menstrual cycle irregularity and risk for future cardiovascular disease. J Clin Endocrinol Metab. 2002;87(5):2013-2017.

2. Solomon CG, Hu FB, Dunaif A, et al. Long or highly irregular menstrual cycles as a marker for risk of type 2 diabetes mellitus. JAMA. 2001;286(19):2421-2426.

3. Bleil ME, Bromberger JT, Latham MD, et al. Disruptions in ovarian function are related to depression and cardiometabolic risk during premenopause. Menopause. 2013;20(6):631-639.

4. Wise LA, Mikkelsen EM, Rothman KJ, et al. A prospective cohort study of menstrual characteristics and time to pregnancy. Am J Epidemiol. 2011;174(6):701-709.

5. Wesselink AK, Wise LA, Hatch EE, et al. Menstrual cycle characteristics and fecundability in a North American preconception cohort. Ann Epidemiol. 2016;26(7):e481:482-487.

6. Murray CJ, Atkinson C, Bhalla K, et al. The state of US health, 19902010: burden of diseases, injuries, and risk factors. JAMA. 2013;310(6): 591-606.

7. Kessler RC, Mcgonagle KA, Zhao S, et al. Lifetime and 12-month prevalence of DSM-III-R psychiatric disorders in the United States. Results from the National Comorbidity Survey. Arch Gen Psychiatry. 1994;51(1):8-19.

8. Vreeburg SA, Hoogendijk WJ, van Pelt J, et al. Major depressive disorder and hypothalamic-pituitary-adrenal axis activity: results from a large cohort study. Arch Gen Psychiatry. 2009;66(6):617-626.

9. Burke HM, Davis MC, Otte C, Mohr DC. Depression and cortisol responses to psychological stress: a meta-analysis. Psychoneuroendocrinology. 2005;30(9):846-856.

10. Young EA, Abelson JL, Cameron OG. Effect of comorbid anxiety disorders on the hypothalamic-pituitary-adrenal axis response to a social stressor in major depression. Biol Psychiatry. 2004;56(2):113-120.

11. Breen KM, Billings HJ, Wagenmaker ER, Wessinger EW, Karsch FJ. Endocrine basis for disruptive effects of cortisol on preovulatory events. Endocrinology. 2005;146(4):2107-2115.

12. Barron ML, Flick LH, Cook CA, Homan SM, Campbell C. Associations between psychiatric disorders and menstrual cycle characteristics. Arch Psychiatr Nurs. 2008;22(5):254-265.

13. Harlow BL, Cohen LS, Otto MW, Spiegelman D, Cramer DW. Early life menstrual characteristics and pregnancy experiences among women with and without major depression: the Harvard study of moods and cycles. J Affect Disord. 2004;79(1-3):167-176.
14. Schliep KC, Mumford SL, Vladutiu CJ, et al. Perceived stress, reproductive hormones, and ovulatory function: a prospective cohort study. Epidemiology. 2015;26(2):177.

15. Ernst CL, Goldberg JF. The reproductive safety profile of mood stabilizers, atypical antipsychotics, and broad-spectrum psychotropics. J Clin Psychiatry. 2002;63 Suppl 4:42-55.

16. Joffe H, Hayes FJ. Menstrual cycle dysfunction associated with neurologic and psychiatric disorders: their treatment in adolescents. Ann NY Acad Sci. 2008;1135:219-229.

17. Wise LA, Rothman KJ, Mikkelsen EM, et al. Design and conduct of an internet-based preconception cohort study in North America: Pregnancy Study Online. Paediatr Perinat Epidemiol. 2015;29(4):360-371.

18. Bech P. Quality of life instruments in depression. Eur Psychiatry. 1997;12(4):194-198.

19. Bech PER, Wermuth L. Applicability and validity of the Major Depression Inventory in patients with Parkinson's disease. Nord J Psychiatry. 1998;52(4):305-310.

20. Bech P, Rasmussen NA, Olsen LR, Noerholm V, Abildgaard W. The sensitivity and specificity of the Major Depression Inventory, using the Present State Examination as the index of diagnostic validity. $J$ Affect Disord. 2001;66(2-3):159-164.

21. Olsen LR, Jensen DV, Noerholm V, Martiny K, Bech P. The internal and external validity of the Major Depression Inventory in measuring severity of depressive states. Psychol Med. 2003;33(2):351-356.

22. Cohen S, Kamarck T, Mermelstein R. A global measure of perceived stress. J Health Soc Behav. 1983;24:385-396.

23. Lee EH. Review of the psychometric evidence of the perceived stress scale. Asian Nurs Res. 2012;6(4):121-127.

24. Cohen $S$. Perceived stress in a probability sample of the United States. In: Spacapan S, Oakamp S, editors. The Social Psychology of Health. Thousand Oaks, CA: Sage Publications; 1988:31-67.

25. Hahn KA, Wise LA, Riis AH, et al. Correlates of menstrual cycle characteristics among nulliparous Danish women. Clin Epidemiol. 2013;5:311-319.

26. Liu Y, Gold EB, Lasley BL, Johnson WO. Factors affecting menstrual cycle characteristics. Am J Epidemiol. 2004;160(2):131-140.

27. Kessler RC, Berglund P, Demler O, et al. The epidemiology of major depressive disorder: results from the National Comorbidity Survey Replication (NCS-R). JAMA. 2003;289(23):3095-3105.

28. Ainsworth BE, Haskell WL, Whitt MC, et al. Compendium of physical activities: an update of activity codes and MET intensities. Med Sci Sports Exerc. 2000;32(9 Suppl):S498-S516.

29. Mckinnon CJ, Hatch EE, Rothman KJ, et al. Body mass index, physical activity and fecundability in a North American preconception cohort study. Fertil Steril. 2016;106(2):451-459.

30. Liang K-Y, Zeger SL. Longitudinal data analysis using generalized linear models. Biometrika. 1986;73(1):13-22.

31. Young EA, Korszun A. The hypothalamic-pituitary-gonadal axis in mood disorders. Endocrinol Metab Clin North Am. 2002;31(1): $63-78$.

32. Butler AC, Chapman JE, Forman EM, Beck AT. The empirical status of cognitive-behavioral therapy: a review of meta-analyses. Clin Psychol Rev. 2006;26(1):17-31. 


\section{Supplementary material}

Table SI Cross-sectional analysis of depression, stress, anxiety, and psychotropic medication use in relation to prevalence of long bleed length ( $\geq 5$ days), PRESTO, 2013-2018

\begin{tabular}{|c|c|c|c|}
\hline & $\begin{array}{l}\text { Long bleeds/ } \\
\text { Total }\end{array}$ & $\begin{array}{l}\text { Unadjusted PR } \\
(95 \% \mathrm{Cl})\end{array}$ & $\begin{array}{l}\text { Adjusted }^{a} \text { PR } \\
(95 \% \mathrm{Cl})\end{array}$ \\
\hline \multicolumn{4}{|l|}{ MDI score } \\
\hline$<20$ & $2030 / 4,388$ & Reference & Reference \\
\hline $20-24$ & $214 / 410$ & $1.13(1.02-1.25)$ & $1.07(0.97-1.18$ \\
\hline $25-29$ & $144 / 272$ & $1.15(1.02-1.29)$ & $1.09(0.97-1.22$ \\
\hline$\geq 30$ & $190 / 396$ & $1.04(0.93-1.16)$ & $0.95(0.85-1.06)$ \\
\hline \multicolumn{4}{|l|}{ PSS-10 score } \\
\hline$<15$ & $926 / 2,073$ & Reference & Reference \\
\hline $15-19$ & $811 / 1,703$ & $1.06(0.99-1.14)$ & $1.05(0.98-1.12$ \\
\hline $20-24$ & $528 / 1,097$ & $1.08(1.00-1.16)$ & $1.03(0.95-1.12$ \\
\hline$\geq 25$ & $313 / 593$ & $1.18(1.08-1.29)$ & $1.10(1.00-1.21)$ \\
\hline \multicolumn{4}{|c|}{ Psychotropic medication use } \\
\hline Never & $\mathrm{I}, 860 / 3,962$ & Reference & Reference \\
\hline Former & $419 / 864$ & $1.03(0.96-1.12)$ & $0.99(0.88-1.1 I)$ \\
\hline Current & $299 / 640$ & $0.99(0.91-1.09)$ & $0.95(0.84-1.08)$ \\
\hline \multicolumn{4}{|c|}{ MDI score and medication history } \\
\hline $\mathrm{MDI}<25$, never treated & $\mathrm{I}, 679 / 3,613$ & Reference & Reference \\
\hline MDI $<25$, formerly treated & $342 / 708$ & $1.04(0.96-1.13)$ & $1.04(0.96-1.13)$ \\
\hline MDI $<25$, currently treated & $223 / 477$ & $1.00(0.91-1.11)$ & $1.01(0.91-1.12)$ \\
\hline $\mathrm{MDI} \geq 25$, never treated & $181 / 349$ & $1.12(1.00-1.24)$ & $1.03(0.93-1.15)$ \\
\hline MDI $\geq 25$, formerly treated & $77 / 156$ & $1.06(0.90-1.25)$ & $0.98(0.84-1.16)$ \\
\hline MDI $\geq 25$, currently treated & $76 / 163$ & $1.00(0.85-1.19)$ & $0.96(0.8 \mathrm{I}-\mathrm{I} .13)$ \\
\hline \multicolumn{4}{|c|}{ PSS-I 0 score and medication history } \\
\hline PSS $<20$, never treated & $\mathrm{I}, 357 / 2,970$ & Reference & Reference \\
\hline PSS $<20$, formerly treated & $227 / 489$ & $1.02(0.92-1.13)$ & $\mathrm{I} .03(0.93-1.14)$ \\
\hline PSS $<20$, currently treated & $153 / 317$ & $1.06(0.94-1.19)$ & $1.09(0.96-1.24)$ \\
\hline PSS $\geq 20$, never treated & $503 / 992$ & $1.11(1.03-1.19)$ & $1.09(0.99-1.20)$ \\
\hline PSS $\geq 20$, formerly treated & $192 / 375$ & $1.12(1.01-1.25)$ & I.II (0.97-I.27) \\
\hline PSS $\geq 20$, currently treated & $146 / 323$ & $0.99(0.87-1.12)$ & $0.99(0.97-1.02)$ \\
\hline
\end{tabular}

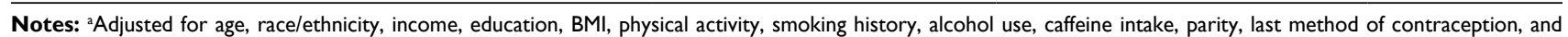
history of anxiety and/or depression diagnosis (model for psychotropic medication use only).

Abbreviations: BMI, body mass index; MDI, Major Depression Inventory; PR, prevalence ratios; PRESTO, Pregnancy Study Online; PSS, Perceived Stress Scale.

\section{Publish your work in this journal}

Clinical Epidemiology is an international, peer-reviewed, open access, online journal focusing on disease and drug epidemiology, identification of risk factors and screening procedures to develop optimal preventative initiatives and programs. Specific topics include: diagnosis, prognosis, treatment, screening, prevention, risk factor modification,

Submit your manuscript here: https://www.dovepress.com/clinical-epidemiology-journa

\section{Dovepress}

systematic reviews, risk and safety of medical interventions, epidemiology and biostatistical methods, and evaluation of guidelines, translational medicine, health policies and economic evaluations. The manuscript management system is completely online and includes a very quick and fair peer-review system, which is all easy to use. 